Abilene Christian University

Digital Commons @ ACU

$9-27-2020$

Social Work Research and Evidence-Based Practice in

Experimental Medicine Exploring Issues in the

Xenotransplantation Context

Alan Lipps

Kyeonghee Jang

Follow this and additional works at: https://digitalcommons.acu.edu/social_work

Part of the Social Work Commons 
Social work research and evidence-based practice in experimental medicine: exploring issues in the xenotransplantation context

\author{
Alan J. Lipps ${ }^{\text {a* }}$ and Kyeonghee Jang \\ ${ }^{a}$ School of Social Work, Abilene Christian University, Abilene, TX, USA; ${ }^{a}$ School of \\ Social Work, Abilene Christian University, Abilene, TX, USA \\ Please direct all correspondence to *Alan J. Lipps, Ph.D., School of Social Work, \\ Abilene Christian University, ACU Box 27866, Abilene, TX 79699-7866, 325-674- \\ 2072, alan.lipps@acu.edu
}

Provide short biographical notes on all contributors here if the journal requires them. 


\title{
Social work research and evidence-based practice in experimental medicine: exploring issues in the xenotransplantation context
}

\begin{abstract}
This paper discusses issues pertaining to the use of evidence-based-practice (EBP), by an interdisciplinary team (IDT), in an experimental medical context. A five-step EBP process originated in medicine and was adapted for use by social work and several other professional disciplines. This paper reviewed EBP terminology, and discussed the meaning and function of evidence-based social work practice within an IDT in a xenotransplantation (XTx) setting. Metaanalytic systematic reviews, and psychosocial surveys, were discussed to glean insights into ways in which IDTs can incorporate those research methodologies into EBP within an experimental medical (i.e., XTx) context. Several issues that arise while conducting psychosocial research in preparation for clinical trials were also discussed. An evidence-based pathway model for interdisciplinary care was proposed and briefly illustrated as a framework for collaboration among IDT members using EBP in XTx practice.
\end{abstract}

Keywords: evidence-based-practice; interdisciplinary teams; xenotransplantation; social work, experimental medicine

Subject classification codes: evidence-based-practice; interdisciplinary teams; xenotransplantation; social work, experimental medicine

\section{Title: Social work research and evidence-based practice in experimental medicine: exploring issues in the xenotransplantation context}

Xenotransplantation, involving the use of genetically-modified animal organs for transplantation into humans (hereafter referred to as XTx), is an experimental surgical procedure in a pre-clinical trial phase of development. Social workers can assume valuable roles as members on interdisciplinary XTx teams. As members of interdisciplinary teams (IDT), social workers also play key roles in other areas of experimental medicine (Walters \& Hurst, 2020). However, interdisciplinary settings in medicine are often dominated by medical professionals whose understanding of the 
scope of social work practice can differ from that of social workers (Cowles \& Lefcowitz, 1992). Recently, Walters and Hurst (2020) urged the social work profession to prepare for advancements in medical technology, such as XTx using genetically modified organs, by clearly articulating the roles social workers can assume in this area of practice.

The purpose of this paper is to discuss issues pertaining to interdisciplinary evidence-based social work practice within a context of XTx, and the broader context of experimental medicine. Specifically, the paper examines: 1) confusion about terminology associated with evidence-based-practice; 2) the use of the evidence-based practice model for social workers employed in experimental medical settings; 3 ) the importance of meta-analytic systematic reviews for evidence-based practice; 4) differences between narrative and systematic reviews of literature; 5) methodological problems within existing psychosocial research that prevent systematic synthesis using meta-analysis; and 6) challenges of psychosocial studies in experimental medicine. The paper concludes with a discussion of how social workers can help interdisciplinary teams (IDT), in experimental medicine, implement EBP collaboratively.

\section{Background}

Traditionally, social workers, working in interdisciplinary, experimental medical contexts focus on providing direct biopsychosocial care for the individual client (Walters \& Hurst, 2020). Walters and Hurst (2020) exclaimed that social workers have skills that allow them to assume much broader functions on interdisciplinary teams (IDTs). They were referring to the person-in-environment perspective that guides social work education and practice. From this perspective, social workers assume dual responsibilities for caring for the well-being of the individual client and the larger social system. As the Code of Ethics of the National Association of Social Workers (2017) 
states: "A historic and defining feature of social work is the profession's focus on individual well-being in a social context and the well-being of society" (Preamble). Social workers, in addition to providing traditional direct-care services, are responsible for ensuring that the interdisciplinary XTx treatment team does no harm to, and promotes the betterment of, society.

In addition, social workers trained to use evidence-based practice (EBP) can offer their unique person-in-environment perspective to the interdisciplinary team (IDT) as they work collaboratively toward providing high quality, evidence-based, interdisciplinary care. EBP was defined by Sackett, Rosenberg, Gray, Haynes and Richardson (1996) as "the conscientious, explicit, and judicious use of current best evidence in making decisions about the care of individual patients" (p. 71). Operationally, the EBP process includes the following five steps:

Step 1 - converting the need for information (about prevention, diagnosis, prognosis, therapy, causation, etc.) into an answerable question... Step 2 - tracking down the best evidence with which to answer that question... Step 3-critically appraising that evidence for its validity (closeness to the truth), impact (size of the effect), and applicability (usefulness in our clinical practice)...

Step 4 -integrating the critical appraisal with our clinical expertise and with our patient's unique biology, values, and circumstances...

Step 5-evaluating our effectiveness and efficiency in executing steps 1 to 4 and seeking ways to improve them both for next time (Straus, Glasziou, Richardson, \& Haynes, 2018 and Haynes, 2019, pp. 4-5)

However, the ability to apply this model in interdisciplinary practice is challenging. Because IDTs have, by definition, practitioners from varying disciplines and 
perspectives, achieving unity or harmony while attempting to apply EBP can be challenging. IDT practitioners have received different training, from different theoretical or conceptual frameworks, using different vocabulary (Satterfield et al., 2009). Without an intentional focus on, and without spending time developing and learning, a common language, a common set of policies, and a common set of practices, there is danger of each team member simply doing their own thing (Bronstein, 2003; Dillenburger et al., 2014; Satterfield et al., 2009). As Dillenburger, Rottgers, Dounavi, Sparkman, Keenan, Thyer, and Nikopoulos, (2014) so eloquently stated, "Without a common conceptual foundation there is a danger that multidisciplinarity regresses into mere eclecticism" (p. 98).

\section{Confusion about terminology associated with evidence-based-practice}

The social work discipline has experienced continuous confusion over the meaning of evidence-based-practice (EBP) due to the overlap between the EBP process and the relatively recent proliferation of evidence-supported practices (Thyer \& Pignotti, 2011). Although early developers and adopters (L. Gibbs \& Gambrill, 2002; L. E. Gibbs, 2003; Regehr, Stern, \& Shlonsky, 2007; Rubin, 2007a; Sackett et al., 1996; Sackett, Straus, Richardson, Rosenberg, \& Haynes, 2001; Shlonsky \& Stern, 2007; Thyer, 2002, 2007) use of the term EBP clearly meant the application of a process oriented EBP model, funding entities tend to use the term evidence-based-practices (i.e., EBPs) to refer to empirically-supported treatments (ESTs), or research supported treatments (RSTs). In addition, the existence of digital repositories of "evidence-based practices" (e.g., John Hopkins University, n.d.; Substance Abuse and Mental Health Services Administration, 2020) can incline some to reason that EBP means simply choosing one of these RSTs, from such a repository, and implementing that practice. While ESTs or RSTs may in fact be identified through the use of the EBP process, confusion arises when ESTs or 
RSTs are mistakenly believed to have the same meaning as EBP (Parrish, 2018; Thyer \& Pignotti, 2011).

\section{Evidence-based practice in interdisciplinary experimental medical settings}

Adding to the confusion regarding EBP terminology, the term practice is not well conceptualized when EBP is used within an interdisciplinary context. Traditionally, social work practice in the medical field involves providing direct psychosocial services, or providing multiple kinds of as-needed assistance to members of the interdisciplinary healthcare team (Browne, 2006; Steketee, Ross, \& Wachman, 2017). In such settings, medical discourse tends to dominate making the focus of IDT communication problem-focused or disease-centric (Giles, 2016). Social work roles and boundaries are not often well-defined and the person-in-environment and strengthsperspectives can be lost (Giles, 2016; Steketee et al., 2017). Other members of the IDT may not understand the purpose or value of having a social worker as a member of the team (Steketee et al., 2017). Perhaps this lack of appreciation is due in part to the fact that social workers can assume multiple roles, and practice a variety of interventions across multiple systems (Steketee et al., 2017).

\section{Social work roles in experimental medicine}

Medical social workers are employed in hospitals, outpatient clinics, dialysis clinics, and in a wide range of other specialized medical settings (Browne, 2006). Social workers frequently serve as valuable members of IDTs in medical settings (Sommers, Marton, Barbaccia, \& Janeane, 2000; Steketee et al., 2017). A recent systematic review (Steketee et al., 2017) indicated that medical social work, in general, is related to improved health-related and cost-containment outcomes. However, this review also highlighted the fact that such research is characterized by heterogeneity of interventions 
and outcomes. Such is due to the diversity of roles that social workers play in healthcare settings. Many of the studies that do exist fail to specify the intervention and outcomes vary widely. Some outcomes are related to patient health, others are related to financial matters, and others pertain to a host of psychosocial variables (Steketee et al., 2017).

Walters and Hurst (2020) believe that the role of social workers working within xenotransplantation (XTx) should expand from the traditional focus on individual patients to include concern for the greater good of society. As the Code of Ethics of the National Association of Social Workers (2017) states: “A historic and defining feature of social work is the profession's focus on individual well-being in a social context and the well-being of society" (Preamble). In essence, social workers, in addition to providing traditional direct-care services, become responsible for ensuring that the XTx treatment team does no harm to, and promotes the betterment of, society.

Another role social workers, on XTx IDTs assume, is that of researcher (Mitchell et al., 2020; Ríos, 2020). Though outside of the scope of the five-steps of EBP, the EBP model assumes practitioners are aware of client preferences, client values, and other contextual variables (Regehr et al., 2007). Additionally, patients enrolled in clinical trials, and in particular clinical trials involving animal-to-human organ transplant, will likely have numerous questions, psychosocial issues, and ethical concerns that will need to be addressed. Therefore, another key role for social workers on XTx transplant teams is that of researcher. Specifically, social workers conduct survey research to learn characteristics of the population to which the experimental treatment will be applied. That research forms the knowledge base by which the XTX IDT is aware of client preferences, values, concerns, etc. Such research can also help social workers be better prepared to help patients' process questions, help patients cope, and deal with the numerous ethical situations that could arise (Walters \& Hurst, 2020). 


\section{What is the social work intervention in experimental medicine?}

The evidence-based-practice (EBP) model was originally designed for medical practice. As such, the model was specifically designed to incorporate evidence of effective treatments for specific medical conditions in decisions regarding the best treatment approach for a particular patient. Although this model was adapted for use by social workers to use in intervening with multiple types of problems (L. Gibbs \& Gambrill, 2002; L. E. Gibbs, 2003; Regehr et al., 2007; Shlonsky \& Gibbs, 2004), in interdisciplinary medical settings confusion can arise over which adaptation of the EBP model to use (Satterfield et al., 2009). Experimental medicine is concerned primarily with testing medical treatments (i.e., transplant surgery, new medications, etc.) to determine the effects they have on medical outcomes (e.g., survival rates). In experimental medicine, the medical problem and intervention are clear; however, that problem and intervention may not be so clear in terms of social work practice. Clearly, the physician, in a XTx context is addressing the problem of organ failure, and intervening by performing transplant surgery. Clearly, the original evidence-based medicine (Sackett et al., 2001) model is the best model for the physician to use to address this problem.

For social workers, who work in experimental medicine, who seek to employ the EBP model, the task of identifying interventions and outcomes gets complicated. A few questions that arise as the practitioner considers using the EBP process include: What are the social work treatments, interventions, and outcomes within experimental medicine? Do social workers, in experimental medical settings, use interventions that are separate from, or different from, interventions offered by physicians and other medical personnel? and, What outcomes are expected following social work interventions? The diverse nature of social work makes clear, precise, operationalizations of interventions and outcomes difficult. Within medical settings, 
social workers provide a diverse array of services including assessment, counseling, information, support, and a host of other services that could be classified as psychosocial interventions (Browne, 2006). However, such services (or interventions) are seldom studied, within the context of experimental medicine, to determine whether they are effective.

\section{Best evidence for evidence-based practice}

In addition to the confusion surrounding the definition of EBP and the definition of social work practice within interdisciplinary, experimental medicine, identifying best available evidence for social workers to use, can be challenging. As stated above, very little research exists pertaining to social work practice within experimental medicine (Walters \& Hurst, 2020). However, as more social workers in experimental medicine produce research, it will be important for practitioners to appraise or synthesize that research. Additionally, as members of the IDT who are trained to use the EBP model, social workers can collaborate with other members of the team through critical appraisal of existing research. In fact, participation in this effort with other team members, from other professions, is essential for building an interdisciplinary evidence-based practice model (Satterfield et al., 2009). In preparation for clinical trials, for example, social workers can assume key roles in conducting, or appraising, systematic reviews of existing evidence.

\section{Meta-analytic systematic reviews}

Much discussion exists about what constitutes the best evidence for the purpose of using the evidence-based-practice (EBP) model to inform treatment decisions (L. E. Gibbs, 2003; Shlonsky \& Gibbs, 2004; Thyer \& Pignotti, 2011). For clinical questions, systematic reviews with meta-analysis of effects from randomized clinical trials are at 
the top of the evidentiary hierarchy (Borenstein, 2019; Borenstein, Hedges, Higgins, \& Rothstein, 2009; L. E. Gibbs, 2003; McNamara \& Scales, 2011; Thyer \& Pignotti, 2011). There are good reasons why systematic reviews that incorporate meta-analysis should occupy this position at the top of the evidentiary hierarchy. One reason is that meta-analysis provides a quantitative synthesis of any given number of experimental or quasi-experimental studies (Borenstein, 2019; Borenstein et al., 2009; Cooper, Hedges, \& Valentine, 2009 2019; Satterfield et al., 2009; Sauerland \& Seiler, 2005). Another reason is that systematic reviews, unlike their predecessor, the narrative review, follow a prescribed methodology. Typically, this methodology includes a focused research question, methods for identifying articles for the review (e.g., by listing search terms and databases searched), criteria for including and excluding studies, critical appraisal of the included studies, extraction of data from the included studies, and analysis of that data. In short, a quality meta-analytic systematic review includes specific methodology designed to minimize bias and synthesizes the results of multiple randomized clinical trials or other treatment effect studies (Cooper et al., 2009; McNamara \& Scales, 2011; Sauerland \& Seiler, 2005).

In addition to meta-analytic systematic reviews offering high quality evidence to support treatments of various problems, they also offer a time-saving benefit for busy practitioners (Sauerland \& Seiler, 2005). Practitioners, on experimental medicine IDTs, for example, are likely busy and simply do not have time to constantly monitor the volumes of journals for recently published randomized clinical trials. However, authors of meta-analytic systematic reviews perform the meticulous tasks of searching databases for published studies, critically evaluating the quality of those studies, extracting data, calculating effect sizes, inputting effect size data into meta-analytic software, and preparing a report that typically includes a point-estimate of the overall 
treatment effect. Such reports generally include several additional metrics that help the reader understand the broader context of the point-estimate (e.g., the degree of heterogeneity of effect sizes for studies included in the meta-analysis).

\section{Narrative vs. meta-analytic reviews}

Prior to the recent surge in popularity and volume of meta-analytic systematic reviews, the best available evidence for practice was the narrative literature reviews (Borenstein, 2019; Borenstein et al., 2009; Cooper et al., 2009; Duffy, 2005; Sauerland \& Seiler, 2005). Many such reviews exist that strive to appraise a body of research and judge whether that research provides evidence that a treatment is effective. A technique, called vote-counting, is sometimes used to determine whether the weight of the evidence favors or disfavors the effectiveness of an intervention (Borenstein, 2019; Borenstein et al., 2009). With vote counting, a reviewer simply counts the studies that are statistically significant, or not statistically significant, to determine if the bulk of evidence supports, or does not support, the intervention (Borenstein, 2019; Borenstein et al., 2009; Cooper et al., 2009).

One common problem with vote counting is that the count is of results of statistical tests of a null hypothesis. Tests of statistical significance are strongly influenced by sample sizes with larger studies being more likely to have a statistically significant result (Borenstein, 2019; Borenstein et al., 2009; Morrison, 2010). This results in at least two additional problems. First, publication bias can result as researchers may be less likely to submit articles for publication when the significance test fails to reject the null hypothesis (Borenstein, 2019; Borenstein et al., 2009). Publication bias may also result if journals are less likely to accept, for publication, studies that fail to reject the null hypothesis (Borenstein, 2019; Borenstein et al., 2009). Second, studies with small sample sizes, that fail to reject the null hypothesis, may be 
counted as evidence of an ineffective intervention (Borenstein et al., 2009). This is true sometimes of studies with robust effect sizes. In other words, many studies might show a strong effect size, but be counted as evidence against effectiveness of an intervention because the statistical significance test indicated that the null hypothesis should not be rejected.(Borenstein, 2019; Borenstein et al., 2009).

To illustrate, imagine that a researcher is reviewing studies pertaining to the effectiveness of methylphenidate for the treatment of attention deficit hyperactivity disorder (ADHD) in adults. Such a study was, in fact, conducted by (Castells et al., 2011). However, for the sake of argument, imagine further, that the hypothetical researcher was conducting a narrative review and identified only the 11 studies shown in Figure 1. Without using a meta-analytic approach for research synthesis, this hypothetical researcher discovers that, out of the 11 studies reviewed, only five of them were statistically significant. The reviewer would likely conclude that a substantial body of evidence exists that shows that the effectiveness of methylphenidate for the treatment of ADHD is mixed. Using a vote counting approach, the researcher might even conclude that the weight of the evidence (i.e., > 50\% of identified studies) shows that methylphenidate is not an effective treatment for adult ADHD.

\section{[FIGURE 1 NEAR HERE]}

On the other hand, consider a meta-analytic study of the same 11 studies. As Figure 1 illustrates, it is quite possible to have several studies reporting statistically insignificant results but have a statistically significant overall effect size in a metaanalysis. As can be seen in the figure, in six out of the 11 studies, the confidence intervals include the null value (i.e., those that cross zero are not statistically significant). However, it is clear that the confidence interval for the overall effect size 
does not cross zero indicating a statistically significant overall effect (using either the fixed effect or random effects models). This is largely due to the fact that, in the metaanalysis, the sample sizes are added together. Increasing the sample size increases the overall precision of, but not the size of, the estimated effect. As can be seen in Figure 1, effect sizes for the 11 studies vary with two studies having below zero effects. The estimate of the overall effect is .35 using the random model.

Another valuable piece of information that might be missed in a narrative literature review, that can be seen in the forest plot presented in Figure 1, is that there is some heterogeneity in the effect sizes. In fact, the two studies at the top of the forest plot found that the effect of the methylphenidate intervention might be harmful (i.e., a negative effect). Using meta-analysis, it would be possible to identify circumstances under which the intervention produced a positive effect and circumstances under which the intervention produced a negative effect. In fact, Castells et al. (Castells et al., 2011) analyzed this data using dose as a moderating variable finding that effect sizes varied significantly as a function of dose.

\section{What if There are no Systematic Reviews or Randomized Clinical Trials?}

Practitioners must make decisions about interventions to offer even in cases where studies of the effectiveness of an intervention for a given problem do not exist. In the absence of studies using such research methods, practitioners, using the EBP process must choose the best available evidence (Thyer \& Pignotti, 2011). As Thyer \& Pignotti (2011) suggest, the practitioner would descend down the evidentiary hierarchy looking for best available evidence. If no meta analytic systematic reviews exist, and if randomized clinical trials do not exist, the practitioner would look for quasi experimental studies. If quasi-experimental studies are nonexistent, the next best evidence would be pre-experimental studies. Continuing down the evidentiary hierarchy 
the practitioner would next look for single case experimental studies, then correlational studies. Given the absence of any research evidence at all, practitioners must then rely on practice knowledge and sound theory.

\section{Methodological problems within existing psychosocial research}

Survey research, to a large extent, uses correlation and regression methods for data analysis. Additionally, in discussions concerning critical appraisal of evidence, the term correlational is commonly used to describe observational or psychosocial survey research (Rubin, 2007b; Rubin \& Babbie, 2017). While not all correlational research is psychosocial research, and not all psychosocial research is correlational, in this paper psychosocial research is synonymous with correlational research. When the only available research is correlational, confusion could arise when attempting to use this hierarchy. Such is the case with research on XTx using genetically modified organs, as clinical trials have yet to be conducted. Without any existing systematic reviews, meta analyses, randomized clinical trials, controlled experiments, or quasi experiments, a hypothetical practitioner might decide that the best available evidence consisted of correlational studies. Such studies tend to use cross-sectional, correlational, survey designs. Confusion could arise when the practitioner attempts to critically appraise such studies to determine if they provide good or poor quality of evidence. As such studies are not concerned with treatment effects, but are mainly concerned with examining a host of psychosocial variables surrounding XTx, the question becomes: of what do these studies provide evidence?

Obviously, correlational research on psychosocial variables are of a different type than are studies concerned with estimating treatment effects. Therefore, it is illogical to think of psychosocial surveys studies as falling on a hierarchy of evidence regarding effectiveness of interventions. This is not to say that correlational studies that 
are concerned with correlating treatments and effects do not exist. However, in the XTx research, much of the correlational research is concerned only with psychosocial variables (Ríos, 2020). A next logical question the practitioner might ask is: since these studies are not useful as evidence for the effectiveness of the intervention (i.e., XTx), do they have any value at all within evidence-based-practice?

Though, assessing the quality of evidence for treatments is a primary task for those using the EBP process, perhaps psychosocial research evidence can contribute to other parts of the EBP model. Most of the concept models for evidence-based practice include practitioner expertise, client preferences and values, and contextual variables (e. g., Gibbs, 2003; Regehr, Stern, \& Shlonsky, 2007; Sackett et al., 2001; Shlonsky \& Gibbs, 2004). However, little attention has been paid to determining, for example, what kind of expertise practitioners need to be effective. Similarly, there does not appear to be an abundance of thought regarding client values and preferences and how knowledge of those might inform the evidence-based-practitioner. However, such concerns are frequently studied by XTx IDTs using psychosocial surveys and are necessary to establish pre-clinical trial knowledge.

As much survey research is concerned with studying psychosocial variables, this methodology is well suited for studying phenomena such as client preferences, values, attitudes, or the anticipated psychosocial ramifications of interventions (Ríos, 2020). In fact, much existing survey research could be used for these purposes. Research about patient attitudes toward XTx, for example, is used to help practitioners know in advance some patient characteristics that might indicate favorability toward receiving such a transplant (Mitchell et al., 2020; Ríos, 2020; Ríos et al., 2018). In fact, such surveys exist that indicate that some variables are associated with favorability toward XTx while others indicate disapproval. In sum, it seems correlational, psychosocial, survey 
research has a valuable contribution to make to evidence-based-practice; however, this value is based not? on assessing effectiveness of interventions, but on the ability to generate information for other parts of the EBP model. Psychosocial surveys, using correlational data analytic strategies, can provide practitioners with knowledge of potential clients' attitudes, values, preferences, etc. toward a potential treatment.

\section{Problems with existing correlational studies}

While methods for reporting results of systematic reviews and for randomized clinical trials are standardized (e.g., CONSORT, 2010; PRISMA, 2009) to facilitate transparency, critical appraisal, and interpretation, no such standards exist for reporting results of correlational (i.e., psychosocial) studies. This presents problems when practitioners and researchers attempt to synthesize knowledge to understand what is known (Mitchell et al., 2020). Without standards, there is typically a wide range of variability in the methods used. Such variation may consist of differences in types and sizes of samples, differences in variables studied, differences in operational definitions of variables, differences in data analysis methods, and differences in reporting results.

A recent study (Mitchell et al., 2020) showed that studies on psychosocial variables related to attitudes toward XTx were characterized by much methodological variability. Variability existed in the way variables (i.e., independent and dependent) were operationalized, in the characteristics of populations sampled, and in analytic strategies used to assess relationships between variables. In general, survey research on psychosocial variables is frequently plagued by a host of problems including small or non-representative samples, low response rates, and heterogeneity in variable operationalization (Ríos, 2020). Such heterogeneity of methods and variable operationalization is especially problematic because it renders comparison of studies 
difficult or impossible (Ríos, 2020). In a commentary on the Mitchell et al. (2020) study, Rios (2020) stated:

On the one hand there is the low quality of the majority of published studies (selection bias, insufficient samples, low study completion rates, etc.) and on the other hand there is heterogeneity between studies regarding different measurement tools and methodology, which complicates any comparison of data. This generates confusion and lack of reliable information in many cases. Moreover, information is generated that does not adapt to reality, hence what are often contradictory data between different studies are actually representing a bias in some of them documenting inadequate information (p. 2).

To enable useful synthesis of psychosocial research, studies must be methodologically similar enough to allow valid between-study comparisons. When studies are not methodologically similar enough to allow valid between-study comparisons, the task of judging whether between study differences in outcomes are due to true differences or attributable to methodological heterogeneity is impossible (Mitchell et al., 2020; Ríos, 2020).(Mitchell et al., 2020; Ríos, 2020). However, increasing the number of comparable psychosocial studies would require agreement between researchers about methodological choices (e.g., standardization of measurement instruments, adherence to sound sampling strategies, consistency in reporting results, etc.). As noted by Rios (2020), "this is an almost impossible task, since each group continues to use different tools and these are not comparable" (p. 2).

\section{Challenges of psychosocial studies in experimental medicine}

As previously discussed, systematic reviews using meta-analyses are only possible when sufficient high-quality studies are available to analyze. To prepare for clinical trials, IDTs frequently conduct psychosocial studies to assess a target population's opinions about the experimental treatment (e.g., XTx). This kind of research helps the 
team to decide whether a new treatment is technically possible, whether the new treatment is expected to outperform other treatments, and whether the new treatment will proceed into a clinical trial. The following discussion concerns questions or concerns that emerge during the process of conducting psychosocial research.

\section{Relevance of studies of opinions about medical treatments}

In preparation for transplantation clinical trials, much of the research is concerned with identifying psychosocial characteristics of a targeted population. A major question concerns the degree to which a target population is favorable toward an experimental procedure. Many times, opinion and attitude surveys are conducted for the purpose of gaining knowledge and understanding of a target population. Such research may, or may not, be published in an academic journal. Similarly, such research may, or may not, be presented at a professional conference.

A question that arises in conducting this type of research concerns two issues commonly discussed in textbooks about research methods (e.g., Rubin, 2007b; Rubin \& Babbie, 2017). These two issues include novelty in research and premature closure of inquiry. Novelty exists when a line of inquiry is considered new and when unanswered questions still exist. Critics of opinion studies, and of other surveys of attitudes, religious beliefs, and other demographic traits, may believe such research is not novel and not relevant. However, it seems unlikely that sufficient research can ever exist to unequivocally establish that knowledge pertaining to attitudes, opinions and beliefs of an experimental treatment (e.g., XTx) is complete. When a new, more effective, medical treatment replaces an older treatment closure of inquiry makes sense. However, as long as treatments, such as XTx with genetically modified animal organs, are novel, it is logical to believe that psychosocial research on population opinions, beliefs, and attitudes is also novel. Therefore, it seems necessary to continue to conduct this type of 
research. Psychosocial variables (e.g., opinions and attitudes) are dynamic and in need of assessment as treatments continue to evolve and values continue to change over time. Attitudes can change depending on the timing of a survey (e.g., before or after a major social incident such as a pandemic) or with different social contexts (Ríos et al., 2018). (Ríos et al., 2018).

\section{Different perspectives on outcome variables}

A second issue in psychosocial research pertains to outcome variables. Evidence of the effectiveness of a particular treatment approach is assessed using randomized controlled trials (RCTs). A typical RCT uses medically-oriented outcomes measured shortly after the treatment. However, some medical researchers have proposed a need for including longer-term outcomes in RCTs. Some suggest using long-term outcome measures to track psychosocial variables such as quality of life, or length of life following treatment (Neuberger et al., 2017). Others emphasize the importance of tracking ethical concerns over time (Romanos, Romanos, Alqahtani, Alqahtani, \& Javed, 2019 Alqahtani, \& Javed, 2019).

Psychosocial studies (e.g., concerning prospective patient attitudes toward a new medical treatment) can potentially help interdisciplinary experimental teams identify long-term outcome variables that can be tracked as part of a RCT. For example, suppose a psychosocial study indicated that favorability toward XTx was influenced by a partner's attitude toward XTx. A RCT could include measures of favorability toward XTx and partner's attitude toward the XTx to evaluate changes in those variables over time. Results of such an evaluation could then inform future practitioners, using the EBP process, about the need for interventions to address discrepancies that might arise over time between the patient and the partner's attitudes toward the XTx. Psychosocial studies might also reveal that there are religious or ethical variables of concern to a 
target population that potentially supersede conducting the RCT. For example, if the target population strongly believes that XTx will do more damage than good, such as spreading a deadly virus, the team may decide to postpone or cancel the RCT.

\section{Measurement Issues: Validity vs. Agreement}

One of the problems with conducting psychosocial studies on opinions, attitudes, and perceptions about an experimental medical procedure (e.g., XTx) pertains to constructing reliable and valid measurement instruments. Such instruments need to be available for use by different researchers in different contexts so that results of similar studies can be directly compared. However, language and cultural differences across different research contexts require that existing measurement instruments be translated for use within those differing contexts. Languages have many nuances that make word for word translations problematic. Therefore, researchers in different cultural settings must often alter instruments to try to generate questions that will produce reliable responses. To enhance reliability, questions often have to be rephrased and answer choices often have to be rewritten to capture the scope of possible responses.

For example, in one recent study (Paris et al., 2016), a reportedly valid and reliable questionnaire of attitudes toward organ donation and transplantation was altered for use with English speaking nursing students. The original response scale used a three-level scale (yes, neutral, and no). This scale was modified to a five-level scale (from strongly disagree to strongly agree). The rationale for this decision was that the five-level rating scale would add information (Chakraborty, Kundel, Nodine, Narayan, \& Devaraju, 1998 Narayan, \& Devaraju, 1998). In addition, this five-level scale can easily be converted back to a three-level scale. Although such instrumentation changes can potentially alter the ability to compare results from studies using the same scale 
(e.g., as in meta-analysis), this particular change was judged to be benign since scores can easily be converted back to the original metric.

Problems will likely be encountered while converting a measurement instrument developed for use in one language and culture to an instrument that produces reliable and valid results in a different language and culture. Obtaining reliable responses to questions about attitudes can be difficult and highly dependent on question and answer options. For example, one may obtain a different answer depending on the answer choices (e.g., 'unfavorable' vs. 'oppositional'). Stubbs, King, and Stuckler (2014) claim that testing the validity of perception-based measures is notoriously difficult. They acknowledge that some concepts suffer from a lack of viable objective alternatives to measure making subjective indicators necessary. Therefore, researchers should pay careful attention to how concepts vary by context, paying careful attention to the subtle nuances of languages. In addition, researchers should collaborate to develop agreed questionnaires that fit different contexts.

\section{Different Perspectives on Statistical Analysis for Psychosocial Studies}

The fourth issue regards statistical analyses. As step three in the EBP model involves critical appraisal of studies, evaluating statistical methods used in different types of studies becomes very important. Different disciplines tend to differ in how they think about, and go about, the business of producing and evaluating evidence for EBP. However, for IDTs using EBP, agreement about what constitutes quality in the design of a study is essential. Such teams should understand that choosing a statistical procedure and the interpretation of its results is far from clear cut. Choices about statistical methods to use within a particular study can vary with respect to the research design (e.g., experimental, longitudinal, survey, etc.), researcher's perspective (logical positivist, social constructionist, etc.), the type(s) of data being analyzed, the complexity 
of the data, the number of cases within a dataset, and the purpose of the research (e.g., exploratory, descriptive, or explanatory).

Within experimental medicine, for example, research is predominantly conducted in a controlled context (e.g., RCTs); the primary purpose is to infer causality regarding a specific treatment; treatment and control groups are statistically compared; sample sizes are relatively small; and, outcome measures are typically measured as events, frequencies, or scores on a scale. Typically, statistical tests for RCTs will be those that test for a significant difference between the control and treatment conditions at the end of the treatment. Such tests may compare sample means of the two groups (i.e., t-tests, ANOVA, ANCOVA) or produce tests of ratios or proportions (e.g., $X^{2}$, risk ratios, odds ratios, etc.).

On the other hand, psychosocial survey research is normally not as concerned with causal inferences, time-order is somewhat arbitrary, and controlling for threats to internal validity is impossible. Multiple regression is well-suited toward data analysis of psychosocial survey data as the goal of such studies is to identify, and quantify, relationships among multiple variables (Berk, 2004; Rubinfeld, 2000). The many forms of regression analysis are well-suited to the multivariate nature of psychosocial surveys. Regression analysis produces a test of the overall model's ability to explain variation in a response variable as well as test the unique contribution each predictor (or independent) variable makes toward the model's ability to explain variation in the response (or dependent) variable (Pardoe, 2013). As with any statistical test, a tendency exists to pay too much attention to statistical significance and neglect other valuable statistics (Borenstein, 2019; Borenstein et al., 2009; Morrison, 2010). In multiple regression, individual parameter estimates are an indicator of the strength of the association between a predictor variable and the response variable. 


\section{Summary and Discussion}

XTx is a pre-clinical, experimental surgical procedure involving the use of geneticallymodified animal organs for transplantation into humans. Because social workers are, and will continue to be, integral parts of interdisciplinary XTx teams, operationally defining how social workers collaborate with other team members to use EBP is essential. This paper defined and discussed the general model of evidence-basedpractice and discussed challenges to the use of that model within interdisciplinary, experimental medicine. Such challenges include continuing confusion about uses of the term EBP, and the meaning of social work practice within an IDT practicing experimental medicine.

Availability of high-quality evidence is a necessity for the successful implementation of the EBP model. To this end, the paper reviewed the methodology and utility of meta-analytic systematic reviews as key sources of high-quality evidence for EBP. To facilitate IDT collaboration, social workers can contribute by using their EBP training to: 1) assist the team in identifying best-available research evidence; 2) working with team members to critically appraise that evidence; 3 ) helping team members decide how each professional can uniquely contribute toward implementing identified RSTs; and 4) collaborating with team members to evaluate the effectiveness of the intervention.

Psychosocial research using survey methodology is commonly used as a part of assessing cultural opinions, attitudes, and values about an experimental treatment (Mitchell et al., 2020; Ríos, 2020). Therefore, some problems and issues sometimes encountered when conducting pre-clinical trial psychosocial research were discussed. The problems and issues included: deciding when sufficient research has been conducted to formulate a complete understanding of a target populations attitudes and opinions toward an experimental treatment (e.g., XTx); use of psychosocial research to 
prepare for RCTs by identifying long-term outcome variables that can be tracked as part of the RCT; difficulties with comparing and synthesizing study results when measurement instruments have to be altered; and, choosing appropriate statistical procedures to use with psychosocial survey data.

\section{Developing an evidence-based pathway model for interdisciplinary care}

No matter what professional disciplines are involved, making IDTs truly collaborative in their use of the EBP model is challenging (Cowles \& Lefcowitz, 1992; Dillenburger et al., 2014; Giles, 2016; Satterfield et al., 2009). A primary difficulty stems from the reality that interdisciplinary practitioners have different educational backgrounds. These differences in education produce barriers between team members that need to be overcome for the team to function effectively. Such barriers include: differences in professional language making communication difficult; differences in the amount of training they have received in using the EBP process; lack of understanding about the knowledge, skills, and abilities possessed by other members of the team; and lack of training in how to function collaboratively as a member of an IDT (Dillenburger et al., 2014; Satterfield et al., 2009).

Given the original sources clearly describe the application of a "process oriented EBP model" to all kinds of practice questions (Parrish, 2018), we would like to suggest a more general model called the evidence based pathway model for interdisciplinary care. This model embraces shared goals and views the IDT activities as collaborative research and practice activities. The model embraces a broader perspective of EBP by: 1. recognizing that IDT team members bring a unique, but equally valuable, perspective to the team; 2 recognizing that all IDT team members bring their own knowledge, and set of practice skills to the team; 3. emphasizing that all IDT members equally share the 
same purpose and goals; 4.recognizing various forms of research as forms of practice; and, 5. and, emphasizing that all team members participate in research activities.

Social workers can help facilitate this process by using their unique training to enhance group functioning (Giles, 2016). Some evidence indicates that health-related outcomes, and IDT performance, are better when a social worker serves as the team leader (Steketee et al., 2017). Social workers can play lead roles in helping to ensure that different members of the team understand the model and agree to work toward shared goals. Social workers can provide education regarding the diversity of perspectives among team members and ensure members share understanding of the core concepts that are essential to the IDT. In short, social workers can assume responsibility for developing IDT training, and on-going group process coaching, so that all team members communicate. Such training can help maximize the probability that IDT members understand key concepts, policies, and procedures that they will follow.

\section{An Example}

Following is an example of how a team leader in a hospital can use the evidence based pathway model for interdisciplinary care. This model uses the same steps as EBP, but explicitly addresses challenges that are due to the different viewpoints among stakeholders. Before using the specific EBP process, the team members should spend considerable time discussing relevant discipline-specific knowledge. Often, this can be accomplished during team meetings by having a representative from each discipline do a presentation. During this presentation, attention can be given to the similarities and differences in terminology pertinent to the treatments to be implemented by each discipline. Presentations, or training meetings, can also help articulate how each discipline will contribute toward achieving the shared goals. 
Step1-A team leader encourages the IDT to identify information to meet the comprehensive needs of a patient. Each member differentially contributes to the team by identifying the needs of the patient. For example, a physician might identify what medications a patient will take to prepare for surgery; while, a social worker might identify that the patient needs counseling to process the psychosocial trauma of transplant surgery.

Step 2-The team leader encourages the IDT to conduct a search for evidence that is useful to meet the needs of the patient; according to each team member's perspective. Each member contributes to the team by identifying best-available evidence for discipline-specific treatment approaches. For example, a physician might identify RCTs supporting several potential medications a patient could take to prepare for surgery; while, a social worker might identify RCTs on different counseling approaches for treating the psychosocial trauma of transplant surgery

Step 3-To critically appraise the evidence identified, the team leader facilitates open discussion among stakeholders (including the client system) about the evidence that was identified. A representative of the team, or a person from a specific discipline, can broadly overview the evidence, discuss the quality of the evidence, and the anticipated consequences of various practices (e.g., surgery, psychosocial therapy, meeting the financial needs),

Step 4- To help the client make an educated decision about a proposed intervention, the team leader can facilitate open discussion about the potential practices identified in step 3. During this discussion, relevant values (personal, professional, and organizational) can be identified and discussed. For example, a patient being told that XTx is a good choice for their clinical circumstance will 
likely need to discuss religious and personal values pertaining to receiving an animal organ into their own body.

Step 5-The team evaluates the effectiveness and efficiency in executing steps 1 to 4 and seeking ways to improve them both for next time. Additionally, the team can collaborate on an evaluation design to evaluate the effectiveness of the intervention.

Though this model is in a developmental phase, the model outlines bow practitioners from different disciplines can collaborate in the EBP process. By IDT members agreeing to share responsibility for working through the steps of the evidencebased pathway model for interdisciplinary care, collaboratiion can be realized.

Potentially, this model can be used by the IDT for planning and conducting research as well as for identifying and appraising existing research evidence. This model can be a useful tool for facilitating IDT collaboration while conducting evaluation research on existing RSTs identified in steps 1 to 3. 


\section{References}

Berk, R. A. (2004). Regression analysis: A constructive critique: Sage.

Borenstein, M. (2019). Common mistakes in meta-analysis: And how to avoid them: Biostat, Incorporated.

Borenstein, M., Hedges, L. V., Higgins, J. P. T., \& Rothstein, H. R. (2009). Introduction to meta-analysis United Kingdom: John Wiley \& Sons, LTD.

Bronstein, L. R. (2003). A model for interdisciplinary collaboration. Social Work, 48(3), pp. 297-306. doi:https://doi.org/10.1093/sw/48.3.297

Browne, T. A. (2006). Social work roles and health-care settings. In S. Gehlert \& T. A. Browne (Eds.), Handbook of health social work. (pp. 23-42). New York, NY: John Wiley \& Sons Ltd.

Castells, X., Ramos-Quiroga, J. A., Rigau, D., Bosch, R., Nogueira, M., Vidal, X., \& Casas, M. (2011). Efficacy of methylphenidate for adults with attention-deficit hyperactivity disorder: A meta-regression analysis. CNS Drugs, 25(2), pp. 157169. doi:10.2165/11539440-000000000-00000

Chakraborty, D. P., Kundel, H. L., Nodine, C. F., Narayan, T., \& Devaraju, V. (1998). Differential receiver operating characteristic (droc) method. Medical Imaging 1998: Image Processing.

CONSORT. (2010). Consort 2010 statement. Retrieved Date Accessed, 2020 from http://www.consort-statement.org/consort-2010.

Cooper, H., Hedges, L. V., \& Valentine, J. C. (2009). The handbook of research synthesis and meta-analysis, 2nd ed New York, NY: Russell Sage Foundation.

Cowles, L. A., \& Lefcowitz, M. J. (1992). Interdisciplinary expectations of the medical social worker in the hospital setting. Health \& Social Work, 17(1), pp. 57-65. doi:10.1093/hsw/17.1.57

Dillenburger, K., Röttgers, H.-R., Dounavi, K., Sparkman, C., Keenan, M., Thyer, B., \& Nikopoulos, C. (2014). Multidisciplinary teamwork in autism: Can one size fit all? The Australian Educational and Developmental Psychologist, 31(2), pp. 97112. doi:10.1017/edp.2014.13

Duffy, M. E. (2005). Systematic reviews: Their role and contribution to evidence-based practice. Clinical nurse specialist CNS, 19(1), pp. 15-17. doi:10.1097/00002800200501000-00005

Gibbs, L., \& Gambrill, E. (2002). Evidence-based practice: Counterarguments to objections. Research on Social Work Practice, 12(3), pp. 452-476. doi:https://doi.org/10.1177/1049731502012003007

Gibbs, L. E. (2003). Evidence-based practice for the helping professions : A practical guide with integrated multimedia: Brooks/Cole-Thomson Learning.

Giles, R. (2016). Social workers' perceptions of multi-disciplinary team work: A case study of health social workers at a major regional hospital in new zealand. [Article]. Aotearoa New Zealand Social Work Review, 28(1), pp. 25-33. doi:10.11157/anzswj-vol28iss1id113

John Hopkins University. (n.d.). Center for evidence-based practice. Retrieved Date Accessed, 2020 from https://www.hopkinsmedicine.org/evidence-basedpractice/.

McNamara, E. R., \& Scales, C. D. (2011). Role of systematic reviews and meta-analysis in evidence-based clinical practice. Indian Journal of Urology, 27(4), pp. 520524. doi:10.4103/0970-1591.91445 
Mitchell, C., Lipps, A., Padilla, L., Werkheiser, Z., Cooper, D. K. C., \& Paris, W. (2020). Meta-analysis of public perception toward xenotransplantation. Xenotransplantation, $n / a(\mathrm{n} / \mathrm{a}), \mathrm{p}$ e12583. doi:https://doi.org/10.1111/xen.12583

Morrison, J. (2010). A power primer: The insignificance of significance in communication research. Florida Communication Journal, 38(2)

National Association of Social Workers. (2017). Code of ethics. Retrieved from https://www.socialworkers.org/About/Ethics/Code-of-Ethics/Code-of-EthicsEnglish

Neuberger, J. M., Bechstein, W. O., Kuypers, D. R., Burra, P., Citterio, F., De Geest, S., . . Krämer, B. K. (2017). Practical recommendations for long-term management of modifiable risks in kidney and liver transplant recipients: A guidance report and clinical checklist by the consensus on managing modifiable risk in transplantation (commit) group. Transplantation, 101(4S), pp. S1-S56. doi:10.1097/TP.0000000000001651

Pardoe, I. (2013). Applied regression modeling (2nd. ed.) Hoboken: NJ: Wiley.

Paris, W., Jang, K., Colsch, L., Prus, A., Bargainer, R., Nour, B., \& Cooper, D. K. (2016). Psychosocial challenges of xenotransplantation: The need for a multidisciplinary, religious, and cultural dialogue. Xenotransplantation, 23(5), pp. 335-337. doi: https://doi.org/10.1111/xen.12263

Parrish, D. E. (2018). Evidence-based practice: A common definition matters: Taylor \& Francis.

PRISMA. (2009). Prisma statement. Retrieved Date from http://www.prismastatement.org/PRISMAStatement/PRISMAStatement.

Regehr, C., Stern, S., \& Shlonsky, A. (2007). Operationalizing evidence-based practice: The development of an institute for evidence-based social work. [Article]. Research on Social Work Practice, 17(3), pp. 408-416. doi:10.1177/1049731506293561

Ríos, A. (2020). Commentary on the manuscript: 'Meta-analysis of public perception towards xenotransplantation'. Xenotransplantation, $n / a(\mathrm{n} / \mathrm{a})$, p e12588. doi:https://doi.org/10.1111/xen.12588

Ríos, A., Ramis, G., Quereda, J. J., Herrero, J. M., Mendonça, L., Muñoz, A., . . . Parrilla, P. (2018). Impact of 2009 pandemic h1n1 influenza a virus on veterinary students' perception of xenotransplantation. Transplantation Proceedings, 50, pp. 2292-2295. doi:https://doi.org/10.1016/j.transproceed.2018.02.178

Romanos, G. E., Romanos, E. B., Alqahtani, F., Alqahtani, M., \& Javed, F. (2019). "Religious belief": An undervalued ethical inclusion criterion for clinical trials on bone grafting procedures. Journal of religion and health, pp. 1-7. doi:10.1007/s10943-019-00851-5

Rubin, A. (2007a). Improving the teaching of evidence-based practice: Introduction to the special issue. [Symposium]. Special issue, 17(5), pp. 541-634. doi:https://doi.org/10.1177/1049731507300145

Rubin, A. (2007b). Research methods for social work: Cengage Learning.

Rubin, A., \& Babbie, E. R. (2017). Empowerment series: Research methods for social work United States: Cengage Learning.

Rubinfeld, D. L. (2000). Reference guide on multiple regression. Reference manual on scientific evidence, 179 , pp. 425-469.

Sackett, D. L., Rosenberg, W. M., Gray, J. A., Haynes, R. B., \& Richardson, W. S. (1996). Evidence based medicine: What it is and what it isn't. BMJ, 312(7023), pp. 71-72. doi:10.1136/bmj.312.7023.71 
Sackett, D. L., Straus, S. E., Richardson, W. S., Rosenberg, W., \& Haynes, R. B. (2001). Evidence-based medicine : How to practice and teach ebm Edinburgh: Churchill Livingstone.

Satterfield, J., M. , Spring, B., Brownson, R., C. , Mullen, E., J. , Newhouse, R., P. , Walker, B., B. , \& Whitlock, E., P. . (2009). Toward a transdisciplinary model of evidence-based practice. The Milbank Quarterly, 87(2), pp. 368-390. doi:10.1111/j.1468-0009.2009.00561.x

Sauerland, S., \& Seiler, C. M. (2005). Role of systematic reviews and meta-analysis in evidence-based medicine. World Journal of Surgery, 29(5), p 582. doi:10.1001/archinte.160.12.1825

Shlonsky, A., \& Gibbs, L. E. (2004). Will the real evidence-based practice please stand up? Teaching the process of evidence-based practice to the helping professions. Brief Treatment and Crisis Intervention, 4(2), pp. 137-153. doi:10.1093/brieftreatment/mhh011

Shlonsky, A., \& Stern, S. B. (2007). Reflections on the teaching of evidence-based practice. Research on Social Work Practice, 17(5), pp. 603-611. doi:https://doi.org/10.1177/1049731507301527

Sommers, L. S., Marton, K. I., Barbaccia, J. C., \& Janeane, R. (2000). Physician, nurse, and social worker collaboration in primary care for chronically ill seniors. Archives of internal medicine(12), p 1825. doi:10.1001/archinte.160.12.1825

Steketee, G., Ross, A. M., \& Wachman, M. K. (2017). Health outcomes and costs of social work services: A systematic review. American Journal of Public Health, 107, pp. S256-S266. doi:10.2105/AJPH.2017.304004

Straus, S. E., Glasziou, P., Richardson, W. S., \& Haynes, R. B. (2018). Evidence-based medicine e-book: How to practice and teach ebm: Elsevier Health Sciences.

Stubbs, T., King, L., \& Stuckler, D. (2014). Economic growth, financial crisis, and property rights: Observer bias in perception-based measures. International Review of Applied Economics, 28(3), pp. 401-418. doi:https://doi.org/10.1080/02692171.2014.884549

Substance Abuse and Mental Health Services Administration. (2020). Evidence-based practices resource center. Retrieved Date Accessed, 2020 from https://www.samhsa.gov/ebp-resource-center.

Thyer, B. A. (2002). Evidence-based practice and clinical social work. Evidence-Based Mental Health, 5, pp. 6-7. doi:http://dx.doi.org/10.1136/ebmh.5.1.6

Thyer, B. A. (2007). Social work education and clinical learning: Towards evidencebased practice? Clinical Social Work Journal, 35(1), pp. 25-32. Retrieved from https://link.springer.com/article/10.1007/s10615-006-0064-2

Thyer, B. A., \& Pignotti, M. (2011). Evidence-based practices do not exist. Clinical Social Work Journal, 39(4), p 328. Retrieved from https://link.springer.com/article/10.1007/s10615-011-0358-x

Walters, W., \& Hurst, D. J. (2020). Xenotransplantation and the nasw code of ethics. Journal of Evidence-Based Social Work, pp. 1-10. doi:https://doi.org/10.1080/26408066.2020.1758266 
Figure 1. Forest plot of selected studies from Castells et al. (2011) meta-analysis of effects of methylphenidate 